\title{
The optimisation of noninvasive ventilation in amyotrophic lateral sclerosis: a systematic review
}

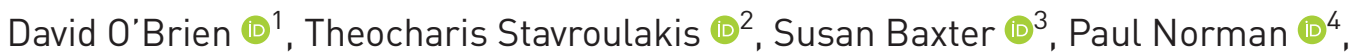 \\ Stephen Bianchi $\mathbb{1}^{5}$, Mark Elliott $\mathbb{1}^{6}$, Maxine Johnson $\mathbb{1}^{3}$, Mark Clowes $\mathbb{1}^{3}$, \\ Aldara Garcia-Sánchez (107), Esther Hobson (10 ${ }^{2,8}$ and Christopher McDermott (10 ${ }^{2,8}$
}

Affiliations: ${ }^{1}$ The Medical School, University of Sheffield, Sheffield, UK. ${ }^{2}$ Sheffield Institute for Translational Neuroscience (SITraN), University of Sheffield, Sheffield, UK. ${ }^{3}$ School of Health and Related Research (ScHARR), University of Sheffield, Sheffield, UK. ${ }^{4}$ Dept of Psychology, University of Sheffield, Sheffield, UK. ${ }^{5}$ Sheffield Teaching Hospitals NHS Foundation Trust, Sheffield, UK. ${ }^{6}$ Leeds Teaching Hospitals NHS Trust, Leeds, UK. ${ }^{7}$ Ramón y Cajal Hospital, Madrid, Spain. ${ }^{8}$ Joint senior authors.

Correspondence: Christopher J. McDermott, Sheffield Institute for Translational Neuroscience, University of Sheffield, Sheffield S10 2HQ, UK. E-mail: c.j.mcdermottdsheffield.ac.uk

@ERSpublications

This systematic review identifies factors associated with the optimal initiation and ongoing monitoring of NIV in patients with ALS. It is recommended to optimise the use of NIV in ALS to improve patient outcomes. http://bit.ly/2L2usJS

Cite this article as: O'Brien D, Stavroulakis T, Baxter S, et al. The optimisation of noninvasive ventilation in amyotrophic lateral sclerosis: a systematic review. Eur Respir J 2019; 54: 1900261 [https://doi.org/ 10.1183/13993003.00261-2019].

\section{ABSTRACT}

Background: Noninvasive ventilation (NIV) prolongs survival and quality of life in amyotrophic lateral sclerosis (ALS); however, its benefits depend upon the optimisation of both ventilation and adherence. We aimed to identify factors associated with effective initiation and ongoing use of NIV in ALS to develop evidence-based guidance and identify areas for further research.

Methods: We searched 11 electronic databases (January 1998 to May 2018) for all types of quantitative and qualitative studies. Supplementary grey literature searches were conducted. Records were screened against eligibility criteria, data were extracted from included studies and risk of bias was assessed. We present findings using a narrative synthesis.

Results: We screened 2430 unique records and included 52 quantitative and six qualitative papers. Factors reported to be associated with NIV optimisation included coordinated multidisciplinary care, place of initiation, selection of interfaces, ventilator modes and settings appropriate for the individual patient, and adequate secretion management. The literature indicated that patients with significant bulbar dysfunction can still derive considerable benefit from NIV if their needs are met. Research emphasises that obstructive airway events, mask leak and uncontrolled secretions should be addressed by adjustments to the interface and machine settings, and the concomitant use of cough augmentation.

Conclusion: This review highlights that NIV optimisation requires an individualised approach to respiratory management tailored to the differing needs of each patient. Ultimately, this should lead to improved survival and quality of life. This review expands on recommendations in current international guidelines for NIV use in ALS and identifies areas for future research. 


\section{Introduction}

In patients with amyotrophic lateral sclerosis (ALS)/motor neurone disease, hypoventilatory respiratory failure is the most common cause of death and respiratory morbidity is related to a poorer quality of life [1]. Noninvasive ventilation (NIV) improves survival and quality of life for patients with ALS in respiratory failure by up to 18 months $[2,3]$. However, the success of NIV is related to its optimisation: the quality (ventilation) and quantity (adherence) of NIV received are prognostic factors in ALS [4].

If ventilation is not optimised the benefits are dramatically reduced: the 1-year survival was $75 \%$ in ALS patients experiencing good correction of hypoxia on NIV, reducing to $43 \%$ in the absence of good correction [4]. Therefore, the effectiveness of ventilation needs to be monitored, with adjustments made to address any issues and compensate for further disease progression. Adherence must also be optimised. Patients who could tolerate NIV for $4 \mathrm{~h} \cdot \mathrm{day}^{-1}$ demonstrated improved survival post-NIV initiation ( 14.2 months) relative to those who used it for $<4 \mathrm{~h} \cdot \mathrm{day}^{-1}$ ( 7.0 months, $\mathrm{p}=0.002$ ) or those who declined NIV (4.6 months, $\mathrm{p}<0.001)$ [5].

NIV is a complex intervention [6], the success of which is influenced by the interaction of multiple service, equipment, patient and carer factors. These may include the service delivery model and process of NIV initiation; the choice of mask interface, ventilator mode and machine settings; and the presence of significant bulbar dysfunction. Guidelines from the National Institute for Health and Care Excellence (NICE) [7], European Federation of Neurological Societies [8] and American Academy of Neurology [9] for the management of NIV in ALS reflect the lack of evidence on how best to address these factors and, consequently, practice varies between services [10]. The aim of this systematic review was to identify factors associated with optimal NIV initiation and ongoing adherence and ventilation in ALS to develop evidence-based guidance and identify areas for further research.

\section{Methods}

\section{Protocol}

The review protocol was registered with the PROSPERO (International Prospective Register of Systematic Reviews) database (www.crd.york.ac.uk/prospero/; registration number CRD42018094394) and was conducted in accordance with PRISMA (Preferred Reporting Items for Systematic Reviews and Meta-Analyses) reporting standards (supplementary appendix 1) [11].

\section{Search strategy}

11 electronic databases were searched (from January 1998 to May 2018, except where stated): MEDLINE via OvidSP, MEDLINE In-Process \& Other Non-Indexed Citations \& Epub Ahead of Print \& MEDLINE without Revisions via OvidSP, Embase via OvidSP, CINAHL via EBSCO, PsycINFO via OvidSP, Cochrane Database of Systematic Reviews via The Cochrane Library (2005 to May 2018), Database of Abstracts of Reviews of Effects via The Cochrane Library (1998 to April 2015; archive only), Cochrane Central Register of Controlled Trials (CENTRAL) via The Cochrane Library, Health Technology Assessment Database via The Cochrane Library, Science Citation Index via Web of Science and Social Sciences Citation Index via Web of Science.

Supplementary searching techniques included hand-searching of included studies' reference lists and grey literature searches using OpenGrey and websites of relevant organisations, including those of the Motor Neurone Disease Association (www.mndassociation.org), NICE (www.nice.org.uk) and NHS Evidence (www.evidence.nhs.uk).

Search terms involved a combination of MeSH subject headings (e.g. "motor neuron disease", "noninvasive ventilation", "artificial respiration") and free-text terms (e.g. ALS, NIV, respiratory failure), with the search strategy developed and led by an information specialist. Searches were limited to studies in humans and presented in the English language. The search strategy for MEDLINE is provided in supplementary appendix 2 .

\section{Study selection}

Citations retrieved from electronic database searches were uploaded to EndNote (version 7; Clarivate Analytics, Philadelphia, PA, USA). Titles and abstracts were independently screened by two reviewers against the eligibility criteria (table 1); a third reviewer resolved any uncertainties. The level of agreement between the two reviewers regarding study inclusion was $>95 \%$. Full texts were then obtained.

\section{Data extraction}

Data were extracted from included studies using a prepiloted extraction form. We collected data on first author, publication year, study design, sample size, population characteristics, data collection method, outcome measures, intervention characteristics, theoretical underpinning, summary of results and main 


\begin{tabular}{|c|c|}
\hline Criterion & Eligibility criteria \\
\hline Population & $\begin{array}{l}\text { Studies in patients with a diagnosis of ALS/MND, or their families and caregivers, or } \\
\text { healthcare professionals involved in their care }\end{array}$ \\
\hline Intervention & $\begin{array}{l}\text { Studies involving any form of long-term, domiciliary NIV, defined as ventilatory support } \\
\text { administered via a removable mask/mouthpiece }\end{array}$ \\
\hline Comparator & Studies with comparator and noncomparator designs \\
\hline Outcome & $\begin{array}{l}\text { Studies reporting any outcome related to the optimisation of NIV initiation, monitoring and } \\
\text { ongoing care (e.g. oxygen and carbon dioxide saturations, patient adherence and } \\
\text { quality-of-life metrics) }\end{array}$ \\
\hline Study design & Empirical quantitative ${ }^{\#}$ and qualitative studies published in English in the past 20 years" \\
\hline \multicolumn{2}{|c|}{$\begin{array}{l}\text { ALS: amyotrophic lateral sclerosis; MND: motor neurone disease; NIV: noninvasive ventilation. " : a range } \\
\text { of quantitative studies were eligible, including randomised controlled trials and other experimental } \\
\text { designs; prospective, retrospective and cross-sectional observational studies; and case studies; " : the } \\
\text { review was restricted to studies published in the past } 20 \text { years to ensure that findings are relevant to } \\
\text { current practice and given potential changes in prognosis during this timeframe. }\end{array}$} \\
\hline
\end{tabular}

author conclusions. The form was suitable for all types of quantitative study designs and was modified for qualitative studies. Extraction forms for each study were completed by one reviewer and verified by a second, as is recommended as an accepted minimum [12]. Multiple citations from a single study were brought together into a single extraction where possible, to avoid double-counting data.

\section{Risk of bias assessment}

We assessed the risk of bias using the established hierarchy of evidence and checklists for each study type, where appropriate. For controlled studies, we considered sources of potential bias as recommended by Cochrane [13]. For other quantitative studies, we used the National Institutes of Health checklists [14]. For qualitative studies, the Critical Appraisal Skills Programme checklist was used [15]. Due to significant between-study heterogeneity, risk of bias was not assessed across the cumulative evidence.

\section{Synthesis}

Study heterogeneity precluded the use of a meta-analysis. Quantitative data were synthesised using a narrative synthesis method. Themes reported in qualitative studies were integrated where they related to the quantitative findings. We brought together studies examining similar processes or reporting similar outcomes, identifying where data agreed and where it conflicted, and provide an indication of the volume and quality of the evidence.

\section{Results}

Study selection

Of 2430 records screened, 221 documents were assessed at full-text level for eligibility, and 58 studies (52 quantitative and six qualitative) were included in the review (figure 1).

\section{Study characteristics}

The included studies involved a range of 1-474 participants (mean 70.69). Five studies used an experimental design: two randomised controlled trials (RCT) $[3,16]$, two randomised crossover studies $[17,18]$ and one quasi-randomised controlled trial [19]. The remaining quantitative studies were observational: 13 prospective, 16 retrospective, seven cross-sectional and 11 case studies. In addition, six qualitative studies were included. Supplementary appendix 3 details the characteristics of included studies.

The included RCTs were found to be at a low risk of bias, while the other three experimental designs had a higher risk of bias, due to issues including unclear random allocation method and reporting bias. The observational studies were all considered to be at higher risk of bias, although some provided more robust evidence than others; for example, seven included studies used a prospective design with sample size $>30$ and adequate control for confounding variables [20-26]. Completed quality assessments for individual studies are available in supplementary appendix 4. 
FIGURE 1 Preferred Reporting Items for Systematic Reviews and Meta-Analyses (PRISMA) flow chart illustrating the study selection process. ": the majority of those excluded on study design were conference abstracts ( $n=80)$ for which no full text could be obtained.

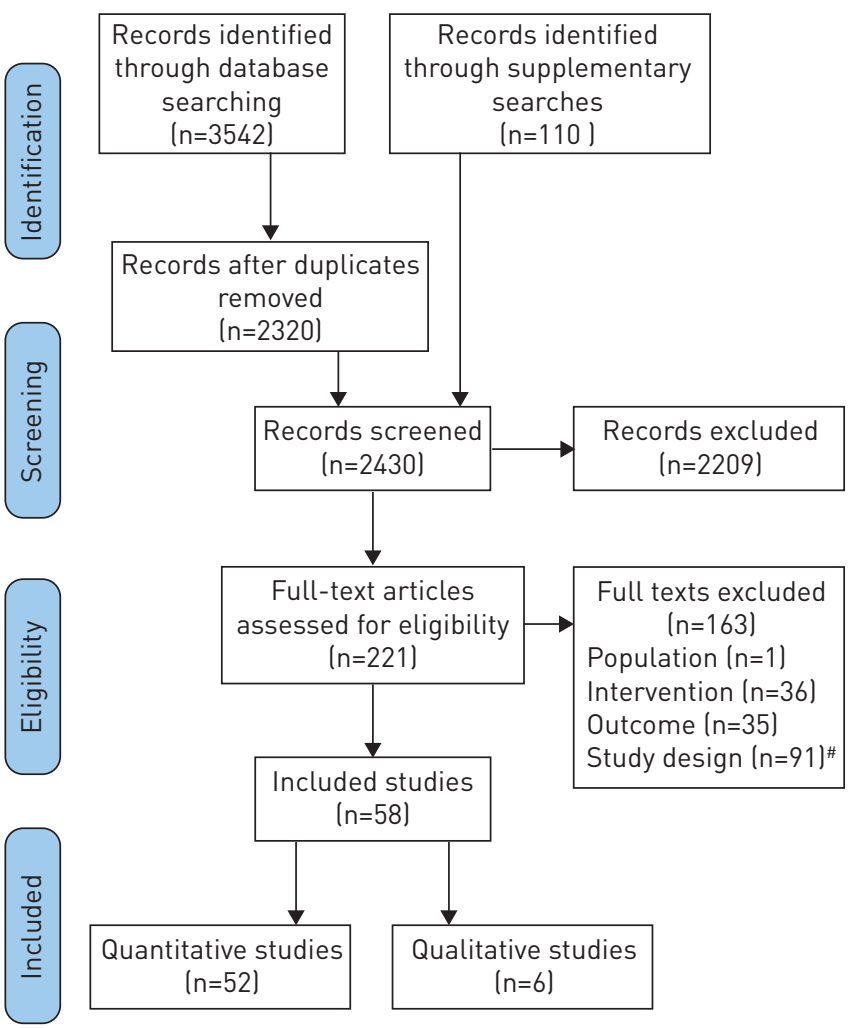

\section{Service factors that influence NIV optimisation \\ Service delivery model}

Eight studies investigated the effects of a multidisciplinary team approach to ALS care on NIV usage: one prospective [27], one retrospective [28] and six cross-sectional studies [29-34]. Evidence was found in all for a positive effect of multidisciplinary care on outcomes related to NIV usage, including access to NIV, patient acceptance and adherence. A prospective Italian study in 37 patients evaluated a complex intervention during the initial adaptation to NIV, involving a patient education session, intensive inpatient monitoring and follow-up with adjustments made as necessary [27]. Adherence $>4 \mathrm{~h} \cdot \mathrm{night}^{-1}$ was achieved in all 37 patients at discharge after a mean \pm SD inpatient stay of $12 \pm 2$ days. At 1 year, 35 (95\%) out of 37 patients remained NIV-adherent. A retrospective before-and-after study in 77 participants observed an increase in NIV acceptance following the addition of a respiratory therapist to the ALS clinic (from 54\% to $82 \%, \mathrm{p}=0.007$ ) [28]. Adherence $>4 \mathrm{~h} \cdot \mathrm{day}^{-1}$ also increased (from $22 \%$ to $73 \%, \mathrm{p}=0.027$ ). Both studies failed to control adequately for confounding variables.

In an epidemiological study of 259 patients in Italy, NIV was more frequently initiated within tertiary ALS centres than general neurology clinics from 1995 to 2004 (37.2\% versus 8.8\%, $\mathrm{p}=0.0001)$ [29]. 76 Italian respiratory centres were surveyed in another study: $90 \%$ used a multidisciplinary approach, involving close cooperation between respiratory and neurology physicians, physiotherapists and psychologists [31]. High-referring centres more frequently reported good collaboration with neurologists than low-referring centres ( $45 \%$ versus $23 \%, \mathrm{p}=0.04)$. Response rates $<50 \%$ increase the risk of bias here. A survey of 11 Canadian ALS specialists cited effective coordination between respiratory and neurology services and the availability of respiratory specialists as contributors to good NIV adherence [30]. A UK audit described the utility of joint respiratory and palliative multidisciplinary clinics for monitoring respiratory function, commencing NIV and discussing end-of-life care in six patients [34].

\section{Place of initiation}

Five studies examined the effects of place of NIV initiation: one RCT [16], one prospective before-and-after study [26], two cross-sectional studies [34,35] and one qualitative study [36]. Two studies evaluated a day case (outpatient) model of initiation $[16,26]$. An Italian RCT compared initiation as a day case $(n=25)$ versus an overnight inpatient $(n=25)$ [16]. Similar rates of NIV adherence were observed during initiation ( $76 \%$ versus $80 \%, \mathrm{p}=0.733)$ and at 3 months ( $68 \%$ versus $76 \%, \mathrm{p}=0.529)$. Patient and healthcare professional satisfaction, respiratory function changes and symptom control were similar in 
both groups. An Australian centre compared a multi-day inpatient initiation $(n=17)$ to a day case initiation $(\mathrm{n}=12)$ in a prospective before-and-after study [26]. "Suitable patients" were initiated as outpatients, highlighting a possible selection bias. Median waiting time for NIV initiation fell from 30 to 13.5 days $(\mathrm{p}<0.04)$, and adverse events (death or acute admission with respiratory failure) declined from four $(24 \%)$ out of 17 to $0(0 \%)$ out of 12 , while daytime arterial carbon dioxide $\left(\mathrm{CO}_{2}\right)$ levels were equivalent. Median post-initiation survival was extended from 278 to 580 days (hazard ratio 0.41, p=0.04).

An international survey of 186 ALS clinicians found that patients are admitted to hospital for initiation more often in Europe than the USA (16 (41.0\%) out of 39 versus 0 (0\%) out of 57, p<0.001) [35]. However, a UK audit described successful home initiation in six patients with the support of a specialist respiratory nurse [34]. A qualitative study reported patient anxiety regarding hospital admission; this was a major barrier for one patient who eventually accepted a trial of NIV as a day case [36].

\section{Equipment factors that influence NIV optimisation Interface}

16 studies examined the effect of the interface on NIV optimisation: one RCT [3], one randomised crossover trial [18], three prospective cohorts [22, 37, 38], four retrospective cohorts [4, 39-41], one cross-sectional survey [30], four case reports [42-45] and two qualitative studies [46, 47]. Interface intolerance was associated with poor adherence in two prospective studies [22,37], one cross-sectional survey [30] and one case report [44]. Commonly reported problems in two qualitative studies included reluctance to use the mask in company, interference with eating and communication, pressure sores, dry mouth, mask leak and claustrophobia, which may contribute to poor adherence [46, 47]. Six studies identified interface-related causes for ineffective ventilation: mask leak in four studies $[4,18,38,41]$ and obstructive sleep apnoea (OSA) in two [39, 42]. A retrospective analysis identified mask leak as a leading source of persistent nocturnal desaturations in $53 \%$ of cases in 82 patients studied [4]. Optimisation of mask fitting successfully minimised leak in this study and a case report [4,45]. In a retrospective cohort, NIV-associated OSA was present in 19 (20.4\%) out of 93 patients using oronasal masks [39]. A case report also described OSA induced by an oronasal mask [42]. Both studies reported changing to a nasal mask with a chin strap to eliminate obstructive events. One higher quality RCT [3], one retrospective cohort [40] and a case report [43] highlighted the value of daytime mouthpiece ventilation, which facilitates adjuvant cough augmentation to expectorate secretions, although this requires adequate bulbar function $[3,40,43]$.

\section{Ventilator type, mode and settings}

18 studies assessed the effect of ventilator type, mode or settings on NIV optimisation: one randomised crossover trial [17], two prospective [37, 38], six retrospective [2, 4, 39, 41, 48, 49], six case reports [42, 45, $50-53]$ and three qualitative studies $[46,54,55]$. A retrospective multicentre cohort with adequate control for confounding variables reported effective ventilation at 1 month in 45 (72.6\%) out of 62 patients using volume-preset (Vol-NIV) systems compared with $40(48.8 \%)$ out of 82 patients using pressure-preset (Pres-NIV) systems $(\mathrm{p}<0.001)$ [2]. However, there were no survival differences between the two groups $(\mathrm{p}=0.533)$. In a retrospective study of 271 patients, average volume-assured pressure support ventilation produced greater average tidal volumes than Pres-NIV (390 versus $356 \mathrm{~mL}, \mathrm{p}=0.007$ ) with similar usage (6.5 versus $6.6 \mathrm{~h} \cdot \mathrm{day}^{-1}, \mathrm{p}=0.703$ ) [49]. These findings are limited by the possibility of selection bias and the use of surrogate outcomes for ventilation (lung volumes) rather than alveolar gases. Three studies reported the effects of switching from Pres-NIV to Vol-NIV or vice versa. Switching from Pres-NIV to Vol-NIV resolved OSA in one patient in a large retrospective study [48] and improved ventilation in another case [44]. A case series described a switch from Vol-NIV to Pres-NIV (in the case of increased leak) and vice versa (in the case of increased secretions), which corrected hypoxia and hypercapnia, and improved symptoms, respectively [51].

The need to make decisions on a case-by-case basis was demonstrated in a randomised crossover trial of spontaneous (S) and spontaneous/timed (ST) NIV modes in 13 patients [17]. The ST mode provided more effective ventilation overall in terms of mean overnight oxygen saturations $(87 \%$ versus $83 \%, \mathrm{p}<0.05)$ and time spent with an overnight transcutaneous $\mathrm{CO}_{2}>55 \mathrm{mmHg}(0 \%$ versus $20 \%, \mathrm{p}<0.05)$; however, four patients (who could not be predicted from baseline characteristics) displayed better ventilation outcomes on the S mode. Survival effects were not assessed, while the risk of bias was considered high due to the small sample size and nonreporting of randomisation processes. Two prospective studies from the same centre described intolerance of the ST mode in seven (20\%) out of 35 patients (five with bulbar involvement) [37] and six (27\%) out of 22 patients (four with bulbar involvement) [38], respectively, which improved upon switching to the $\mathrm{S}$ mode in all cases.

Low-quality evidence from three case reports suggested poor NIV adherence due to air-swallowing [53], excessively high airway pressures on Pres-NIV machines [50] and target tidal volumes on Vol-NIV 
systems [45]. In the latter case, adherence improved when target tidal volumes were reduced from $8 \mathrm{~mL} \cdot \mathrm{kg}^{-1}$ to $6 \mathrm{~mL} \cdot \mathrm{kg}^{-1}$. Three qualitative studies linked discomfort associated with air pressure to reduced adherence $[46,54,55]$. Conversely, insufficient ventilation settings were associated with poor ventilation in $34.7 \%$ patients in one retrospective study [41]. Three studies reported the benefit of increasing ventilator support to improve ventilation, by increasing inspiratory positive airway pressure in a retrospective cohort [4] and case report [45] and increasing target tidal volumes in another case report [51]. Increasing expiratory positive airway pressure helped to eliminate OSA in three retrospective studies $[4,39,48]$. Other successful measures found to improve ventilation are reported in table 2. A flow chart summary can be found in supplementary appendix 5 .

\section{Adjuvant therapies}

Seven studies described the use of adjuvant interventions: two prospective [27, 60], two retrospective [48, 61] and three case studies $[44,50,62]$. Music-assisted relaxation was beneficial in supporting NIV transition within the first week in a prospective feasibility study of 15 patients [60]; however, risk of bias was deemed high due to methodological flaws in participant selection, outcome measurement and statistical analysis. Successful use of a portable hand-held ventilator (Philips "Vitabreath") in three patients was reported in a case series, although the authors indicated that patients may struggle with the pressure differences compared to their usual ventilators [62]. Two studies examined the use of mandibular advancement devices in treating OSA and improving NIV adherence: one case report described its successful use [50] while a retrospective study found no benefit [48].

\section{Secretion management}

Nine studies examined the impact of controlling secretions on NIV optimisation: two prospective [24, 27], three retrospective [56-58], three case reports $[43,44,59]$ and one qualitative study [46]. An association between excess oropharyngeal or airway secretions and poor adherence was found in two prospective studies [24, 27] and one case report [59]. One prospective study found that an absence of airway secretions was predictive of good adherence (odds ratio 11.5, 95\% CI 1.3-98.4), while the case report described an improvement in NIV usage (from $<1 \mathrm{~h} \cdot$ night $^{-1}$ to $6-8 \mathrm{~h} \cdot$ night $^{-1}$ ) following treatment with a subcutaneous glycopyrrolate infusion [59].

Five studies highlighted the role of cough augmentation in the active management of secretions: three retrospective studies [56-58] and two case reports [43, 44]. A retrospective analysis of 474 patients found a significant improvement in survival between NIV users who also used daily cough-assist compared to those using NIV alone (median 25.73 months versus 15.00 months, $\mathrm{p}<0.001$ ) [58]. Combined use of NIV

TABLE 2 Troubleshooting: noninvasive ventilation (NIV) problems and solutions

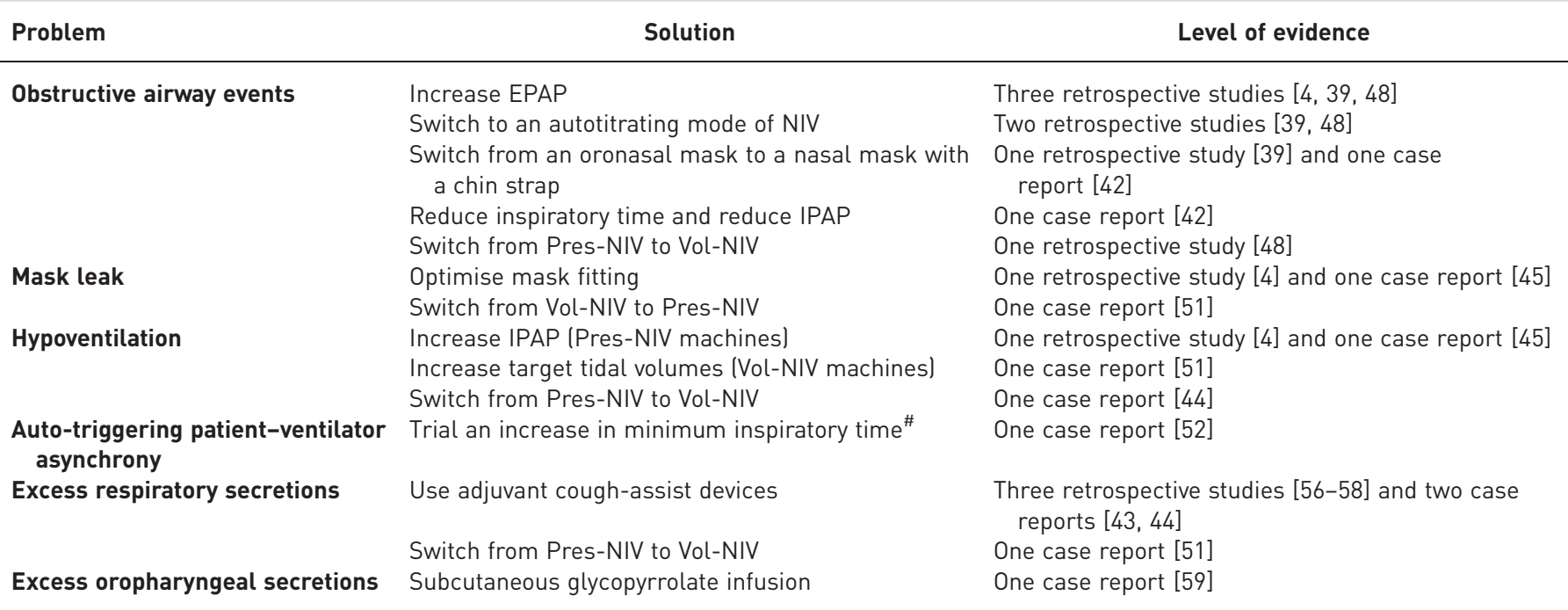

EPAP: expiratory positive airway pressure; IPAP: inspiratory positive airway pressure; Pres-NIV: pressure-preset noninvasive ventilation; Vol-NIV: volume-preset noninvasive ventilation. " : only after first ensuring that the mask and tubing are free from condensation, that unintentional leak is minimised and that appropriate trigger and cycle sensitivities are set. Minimum inspiratory times may not be adaptable on some ventilators. 
and cough-assist was successful in avoiding tracheostomy in two retrospective studies of 101 patients each $[56,57]$ and resolving 43 (55\%) out of 78 desaturation episodes [57].

\section{Efficacy monitoring \\ Monitoring ventilation}

Two retrospective studies reported that effective ventilation predicted survival [4, 48], while another retrospective study did not find the effectiveness of NIV to be a prognostic factor [2]. In one study, only $40(49 \%)$ out of 82 patients were effectively ventilated at 1 month [4]. Importantly, 1-year survival was $75 \%$ in those effectively ventilated at 1 month, declining to $43 \%$ in those ineffectively ventilated $(\mathrm{p}=0.002)$. Remedial measures at 1 and 3 months corrected ventilation by month six in 12 (43\%) patients and 1-year mortality in this subgroup was similar to those effectively ventilated at 1 month (four deaths versus three, $\mathrm{p}=0.13)$. In $16(57 \%)$ patients NIV was still inadequate at month six despite corrective measures. 1-year mortality in this subgroup was significantly higher than in those effectively ventilated at 1 month (seven deaths versus three, $\mathrm{p}=0.002)$. Another study found ineffective ventilation in 73 (41\%) out of 179 patients at 1 month, due to OSA in 49 cases (67\%) [48]. Individuals adequately ventilated at 1 month, and those for whom OSA was successfully eliminated within the first month, experienced greater median survival (26 (13-45) months and 29 (20-53) months, respectively) than those who were inadequately ventilated at 1 month due to uncorrected obstructive events $(14$ (7-27) months, $\mathrm{p}<0.05)$ or other causes $(12(6-23)$ months, $\mathrm{p}<0.05)$.

Two retrospective studies investigated the need for longitudinal adaptations to machine settings with disease progression $[2,61] .28(78 \%)$ out of 36 patients required at least one upward change in pressure settings [61]. In a two-centre comparison, ventilator setting changes were required more frequently in 82 patients using pressure-preset (Pres-NIV) than 62 patients using volume-preset (Vol-NIV) machines: $51 \%$ versus $14 \%(\mathrm{p}<0.001)$ [2]. Of those ineffectively ventilated in the first month, effective ventilation was achieved after the first modification in $79 \%$ of the Vol-NIV group and $31 \%$ of the Pres-NIV group $(\mathrm{p}<0.001)$.

\section{Monitoring adherence}

Survival correlated with hours of NIV use in three papers: one prospective cohort [21] and two retrospective cohorts [5, 58]. 17 studies reported NIV adherence rates: nine prospective [20-24, 27, 37, 38, 63], seven retrospective $[2,4,28,48,49,61,64]$ and one cross-sectional study [29]. The proportion of patients achieving $\geqslant 4 \mathrm{~h}$ of use per day ranged from $46 \%$ to $100 \%$.

\section{Telemonitoring}

The costs of a home telemonitoring system were analysed in a quasi-randomised controlled trial of 39 patients, observing a 55\% reduction in average total costs in patients with the telemonitoring system (EUR 8908.6 \pm 6552.7 ) versus those on standard care (EUR 19664.9 \pm 5256.5 ) due to reduced healthcare utilisation [19]. However, this reduction did not reach statistical significance $(\mathrm{p}=0.058)$. The impact of the telemonitoring system on NIV efficacy or survival was not assessed. This trial was judged to be at high risk of bias due to a nonrandom allocation method and potential reporting bias.

\section{Patient and carer factors that influence NIV optimisation Bulbar dysfunction}

23 studies investigated the effects of bulbar impairment on NIV optimisation: two RCTs [3, 16], 10 prospective cohorts $[20-25,37,38,63,65]$, six retrospective cohorts $[40,56-58,64,66]$, two cross-sectional studies $[29,30]$ and three case reports $[43,44,67] .19$ studies found an association between bulbar impairment and poorer adherence or ventilation, while four studies (including one RCT) identified no such association. In this RCT, bulbar-onset disease predicted greater NIV adherence [16]; however, another RCT reported an average use per day of $9.3 \mathrm{~h}$ in the more preserved bulbar function subgroup versus $3.8 \mathrm{~h}$ in those with poor bulbar function [3]. In two prospective studies of 71 and 73 participants with adequate control for confounding variables, those with more severe bulbar impairment had an increased risk of poor adherence: six-fold in one (odds ratio 6.09, 95\% CI 1.18-31.52) [21] and eight-fold in another (odds ratio 8.5, 95\% CI 1.6-46.2) [24]. In the latter trial, bulbar-predominant patients were reported to need more intensive and prolonged monitoring at NIV onset to maximise adherence [24]. In spite of this, one prospective cohort reported 1-year adherence $>4 \mathrm{~h} \cdot \mathrm{day}^{-1}$ in 35 (95\%) out of 37 patients who had undergone an intensive education and adaptation programme at initiation despite nine presenting severe bulbar involvement at initiation and 23 mild-moderate impairment [27].

18 studies investigated survival or quality-of-life outcomes in patients with bulbar dysfunction using NIV. 14 studies found that bulbar patients derived some benefit from NIV: two RCTs [3, 16], six prospective $[20,21,27,37,38,65]$, five retrospective $[5,41,58,64,68]$ and one cross-sectional study [29]. Four 
studies reported no benefit: three retrospective [40, 56, 57] and one case study [67]. Generally, the gains derived in bulbar patients were reduced compared with nonbulbar patients, as reflected in an RCT where a subgroup with poorer bulbar function experienced no survival gain despite some quality-of-life benefits [3]. However, the trial was not powered for this subgroup analysis. Perhaps surprisingly, one retrospective study observed a median survival benefit from NIV of 13 months in all 219 patients, increasing to 19 months in 58 with bulbar-onset disease [68].

\section{Patient and carer perceptions}

The effect of patient and carer perceptions on the optimisation of NIV was examined in four qualitative studies, involving between five and 37 participants [46, 47, 54, 69]. Adaptation to NIV takes time for many patients, beginning with familiarisation with the equipment [69]. Some patients highlighted the importance of an initial trial period to gauge the positive effects of NIV [69]. Determination and perseverance were required to optimise adherence, while accessible in-person or telephone support might be beneficial in overcoming early obstacles [46]. Carers reported a lack of confidence in adjusting the machine [46], which lessened with increased familiarity [47]. Sleep disturbance may occur in patients and carers due to the machine noise and having to make adjustments to the system $[46,54]$. A positive coping style and perceived need to engage with the treatment was related to better adherence, while feelings of hopelessness were associated with poor adherence [54]. Hopelessness was observed to be modifiable and improvements positively influenced patient attitudes to NIV.

\section{Discussion}

This review highlights the importance of optimising both adherence and ventilation to gain the full benefit from NIV for patients with ALS. Patients must receive effective routine services, which should also be highly adaptable and coordinated to support the most complex patients who require the greatest clinical input. We recommend that services should adopt a coordinated, multidisciplinary approach aligned with current guidelines [7-9], but go further to recommend the need for specific professionals (including respiratory specialists) to be involved early and throughout the disease course to optimise adherence and ventilation. This will allow barriers to successful NIV use to be addressed prior to NIV initiation. The key benefits of this set-up in optimising NIV use may be one explanation for why multidisciplinary care is associated with improved survival compared to nonspecialist services [32, 70-73].

Current guidelines make no reference to place of initiation, leading to variations in practice [7-9]. Outpatient or domiciliary initiation may promote acclimatisation, minimise patient anxiety and reduce delays in commencing NIV. In patients with chronic respiratory failure, outpatient [74] and domiciliary initiation [75] have been comparable to inpatient models in terms of adherence and ventilation and more cost-effective. The American Academy of Sleep Medicine suggest that inpatient initiation can be difficult to justify both medically and financially [76]. The Medical Research Council advises that complex interventions such as NIV work best if tailored and evaluated according to local circumstances rather than being completely standardised [6]. Therefore, we recommend that outpatient (and perhaps home) initiation should be considered as an effective alternative to inpatient initiation; however, there should be means for more complex patients (e.g. those with significant bulbar impairment) to receive greater attention, which could involve an inpatient stay and more intensive monitoring to optimise efficacy.

We have highlighted the importance of the mask interface in NIV success and recommend that interface selection and fitting should be optimised to minimise leak and maximise comfort. This might involve offering a variety of interfaces and providing alternatives as necessary. Particular interfaces will have key benefits in certain scenarios; for instance, switching from an oronasal mask to a nasal mask with a chin strap to address obstructive events and the use of a mouthpiece in patients with adequate bulbar function to allow for daytime use and adjuvant cough augmentation. A recent review highlighted the broad range of airway clearance techniques available, which may be adapted according to individual patient requirements [77]. Secretion management via cough augmentation and various pharmacological methods should be optimised alongside NIV.

It remains unclear which initial ventilator types, modes and settings are optimal, but what is very clear is that setting adjustments are often required to achieve success. Ventilator choice may be restricted within certain health services, while the potentially damaging effects of using a spontaneous mode in patients with progressive respiratory muscle weakness must be considered [76]. Effective ventilation is likely to confer a survival advantage, so we recommend this should be a goal in the first few months post-initiation while maintaining the balance between effective adherence, ventilation and comfort. To achieve this, and in contrast with published guidance, which suggests reviewing patients every 2-3 months [7-9], we recommend reviewing patients in the first few days and weeks to screen for and identify causes of poor efficacy and adjust NIV settings accordingly as adjustments appear to prolong survival [4, 48]. Particular 
TABLE 3 Evidence-based recommendations

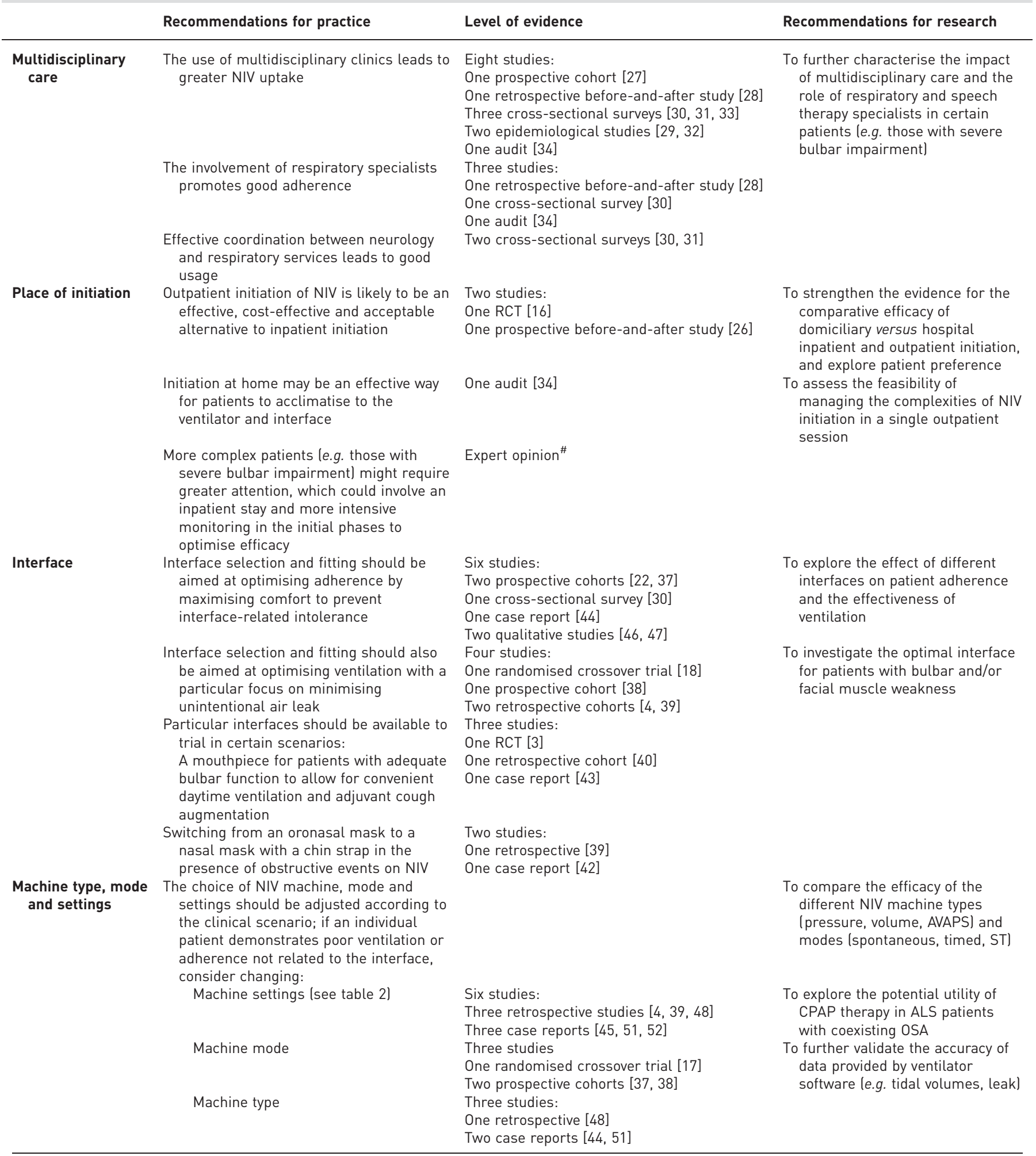


TABLE 3 Continued

\begin{tabular}{|c|c|}
\hline & Recommendations for practice \\
\hline \multirow[t]{2}{*}{$\begin{array}{l}\text { Secretion } \\
\text { management }\end{array}$} & $\begin{array}{l}\text { Cough-assist machines for airway } \\
\text { secretion clearance should be combined } \\
\text { with NIV to maximise survival and } \\
\text { quality-of-life benefits }\end{array}$ \\
\hline & $\begin{array}{l}\text { Ensure optimal oropharyngeal secretion } \\
\text { control prior to and during NIV use to } \\
\text { promote effective ventilation and good } \\
\text { adherence, through the use of various } \\
\text { pharmacological and } \\
\text { nonpharmacological methods }\end{array}$ \\
\hline \multirow[t]{4}{*}{ Monitoring } & $\begin{array}{l}\text { Close monitoring of all patients in the first } \\
\text { few days and weeks post-initiation }\end{array}$ \\
\hline & $\begin{array}{l}\text { Additional provision for those at higher risk } \\
\text { of difficulties (e.g. patients with severe } \\
\text { bulbar impairment) }\end{array}$ \\
\hline & $\begin{array}{l}\text { Monitoring to identify poor adherence and } \\
\text { ineffective ventilation and the } \\
\text { implementation of corrective measures } \\
\text { that could extend survival }\end{array}$ \\
\hline & $\begin{array}{l}\text { Vigilant assessment of the optimal } \\
\text { interface and NIV settings over time in } \\
\text { the context of evolving bulbar } \\
\text { dysfunction and gradual weakening of } \\
\text { the respiratory muscles }\end{array}$ \\
\hline
\end{tabular}

\section{Patient and carer} perceptions

Bulbar impairment

Psychological interventions may be helpful where adherence is suboptimal

Patients with significant bulbar dysfunction
Level of evidence

Five studies:

Three retrospective cohorts [56-58]

Two case reports $[43,44]$

Four studies:

Two prospective cohorts [21, 24]

One case report [59]

One qualitative study [46]

Expert opinion

Expert opinion

Two retrospective studies $[4,48]$

Two retrospective studies $[2,61]$

Expert opinion

One qualitative study [55] can still derive significant benefit from NIV if their needs are met

Measures that should be taken to promote effective NIV use in these patients include:

Multidisciplinary care

Effective secretion management

Optimisation of interface selection and fitting

Initial adaptation, which might involve more intensive inpatient initiation procedures than for those with better bulbar function

Vigilant monitoring and troubleshooting

Proactive management, including

attention to the appropriate interface and machine settings over time
14 studies:

Two RCTs $[3,16]$

Six prospective cohorts $[20,21,27,37,38,65]$

Five retrospective cohorts $[5,41,58,64,68]$

One cross-sectional study [29]

Expert opinion
Recommendations for research

To compare the effectiveness and cost-effectiveness of the various manual and mechanical airway clearance techniques that may be used in ALS

To explore a large-scale, multidisciplinary initiation programme involving education, adaptive measures and monitoring

To explore the most clinically- and cost-effective methods for monitoring the effectiveness of NIV

To explore the best methods for monitoring patients who are deteriorating on NIV

To further investigate the potential utility of telemonitoring of NIV in ALS and resolve the issues with current systems le.g. system specification, pre-determined alerts and the delivery of a patient-specific action plan)

To explore the effects of different psychological interventions on NIV acceptance and adherence in ALS

To further examine the effect of these recommended measures on the efficacy of NIV in patients with significant bulbar dysfunction

NIV: noninvasive ventilation; RCT: randomised controlled trial; AVAPS: average volume-assured pressure support; ST: spontaneous-timed (mode); CPAP: continuous positive airway pressure; ALS: amyotrophic lateral sclerosis; OSA: obstructive sleep apnoea. \# : conclusions drawn by the review authors in their interpretation of the evidence. Some of these conclusions are reflected in the discussion sections of included studies.

attention should be paid to patients at higher risk of difficulties. Clinicians should be vigilant in their assessment of the optimal interface and NIV settings over time, as they will probably need altering due to evolving bulbar dysfunction and gradual weakening of the respiratory muscles. The most appropriate tests to monitor ventilation remain unclear. A working group of 10 European ventilation specialists 
recommended that more complex tests (e.g. polysomnography) could have utility in titrating parameters, troubleshooting for problems and monitoring efficacy in more complex patients, but simple oximetry and ventilator-recorded data may suffice in most cases [78]; this would free up resources to focus on those with the most barriers to success. An RCT in patients using positive airway pressure for OSA found that use of a telemonitoring system that allowed clinicians to identify problems and make adjustments to the settings remotely significantly improved adherence versus those using standard care [79]. A 2016 systematic review recommended that further evidence is needed to demonstrate the value of telemonitoring in ALS [80].

Currently, there is a reluctance among some clinicians to offer NIV to patients with significant bulbar impairment [10] and current guidelines suggest that these patients should only receive a trial of NIV if they are likely to benefit from an improvement in sleep-related symptoms [7], or should instead be offered tracheostomy-assisted ventilation or palliative care [8]. While patients with bulbar dysfunction face greater barriers to NIV success, the evidence suggests that they may gain both symptom and survival benefit that could be at least equal to that of nonbulbar patients. This benefit depends on strategies to promote effective use; for example, attention to secretion management, interface optimisation, adequate initial acclimatisation and ongoing active management with vigilant monitoring and adjustments made as necessary. These complex patients derive the greatest benefit from a multidisciplinary approach [71].

Qualitative studies highlighted the need to identify and address the barriers to acceptance and adaptation of the patient and carer to life with NIV. However, there is a lack of evidence on interventions to support patients and carers. An ongoing RCT in patients with chronic obstructive pulmonary disease is evaluating the effects of counselling, relaxation, mindfulness-based exercises and neuropsychological rehabilitation on NIV acceptance and adherence [81]. One included study suggested that an educational programme can have a significant impact on adherence [27], while another proposed offering psychological interventions where adherence is suboptimal [55].

\section{Strengths and limitations of this review}

This is a systematic examination of the evidence exploring the optimisation of NIV use in ALS. Due to the breadth of the review, reporting has been unable to examine each paper in depth. Studies identified were largely observational with a paucity of randomised controlled trials. Therefore, any conclusions drawn from this review must be interpreted in light of the limited high-quality evidence available. Furthermore, any evidence must be considered in the context of individual patient and service needs. RCT evidence is likely to remain rare in this patient group due to ethical issues and clinical heterogeneity. Nonetheless, the review findings may be used to inform the development of guidelines for optimising the ALS patient care pathway and highlight areas that warrant further research (see table 3).

\section{Conclusion}

There is a substantial body of evidence related to the optimal care of ALS patients on NIV, considering service, equipment, patient and carer factors. Factors optimising care for all patients include effectively coordinated multidisciplinary care; careful selection of interfaces, ventilator modes and settings appropriate for the individual patient; adequate secretion control; and vigilant monitoring and adjustment of settings. Attention to the factors identified will enable the delivery of evidence-based NIV therapy, which should ultimately improve patient survival and quality of life.

Conflict of interest: D. O'Brien reports a bursary from The Wolfson Foundation during the conduct of the study. T. Stavroulakis reports grants from National Institute for Health Research (NIHR) under the Research for Patient Benefit (RfPB) programme during the conduct of the study. S. Baxter has nothing to disclose. P. Norman has nothing to disclose. S. Bianchi has nothing to disclose. M. Elliott reports personal fees from Philips Respironics, ResMed, and Fisher and Paykel, outside the submitted work. M. Johnson has nothing to disclose. M. Clowes has nothing to disclose. A. Garcia-Sánchez has nothing to disclose. E. Hobson reports receiving a grant from the National Institute for Health Research (NIHR) under the Research for Patient Benefit (RfPB) programme; further support was provided by the NIHR Sheffield Biomedical Research Centre (BRC); E. Hobson also received equipment from Philips Respironics as part of a previous research study. C. McDermott reports grants from the National Institute for Health Research (NIHR) under the Research for Patient Benefit (RfPB) programme during the conduct of the study; further support was provided by the NIHR Sheffield Biomedical Research Centre (BRC); C. McDermott is a member of the data safety monitoring board for Orphazyme and Orion Pharma outside of the submitted work, and received equipment from Philips Respironics as part of a prior research study.

Support statement: The study was funded by The National Institute for Health Research (NIHR), within the Research for Patient Benefit Programme (PB-PG-1216-20041) and supported by the NIHR Sheffield Biomedical Research Centre (IS-BRC-1215-20017). E. Hobson is funded by the NIHR and Health Education England (Clinical Lectureship scheme). The views and opinions expressed therein are those of the authors and do not necessarily reflect those of the NIHR, National Health Service (NHS) or the Department of Health and Social Care. Funding information for this article has been deposited with the Crossref Funder Registry. 


\section{References}

1 Rafiq MK, Proctor AR, McDermott CJ, et al. Respiratory management of motor neurone disease: a review of current practice and new developments. Pract Neurol 2012; 12: 166-176.

2 Sancho J, Servera E, Morelot-Panzini C, et al. Non-invasive ventilation effectiveness and the effect of ventilatory mode on survival in ALS patients. Amyotroph Lateral Scler Frontotemporal Degener 2014; 15: 55-61.

3 Bourke SC, Tomlinson M, Williams TL, et al. Effects of non-invasive ventilation on survival and quality of life in patients with amyotrophic lateral sclerosis: a randomised controlled trial. Lancet Neurol 2006; 5: 140-147.

4 Gonzalez-Bermejo J, Morelot-Panzini C, Arnol N, et al. Prognostic value of efficiently correcting nocturnal desaturations after one month of non-invasive ventilation in amyotrophic lateral sclerosis: a retrospective monocentre observational cohort study. Amyotroph Lateral Scler Frontotemporal Degener 2013; 14: 373-379.

5 Kleopa KA, Sherman M, Neal B, et al. Bipap improves survival and rate of pulmonary function decline in patients with ALS. J Neurol Sci 1999; 164: 82-88.

6 Craig P, Dieppe P, Macintyre S, et al. Developing and evaluating complex interventions: the new Medical Research Council guidance. Int J Nurs Stud 2013; 50: 587-592.

7 National Institute for Health and Care Excellence. Motor Neurone Disease: Assessment and Management (NICE Guideline NG42). 2016. www.nice.org.uk/guidance/ng42/

8 Andersen PM, Abrahams S, Borasio GD, et al. EFNS guidelines on the clinical management of amyotrophic lateral sclerosis (MALS) - revised report of an EFNS task force. Eur J Neurol 2012; 19: 360-375.

9 Miller RG, Jackson CE, Kasarskis EJ, et al. Practice Parameter update: The care of the patient with amyotrophic lateral sclerosis: drug, nutritional, and respiratory therapies (an evidence-based review). Neurology 2009; 73: $1218-1226$.

10 O’Neill CL, Williams TL, Peel ET, et al. Non-invasive ventilation in motor neuron disease: an update of current UK practice. J Neurol Neurosurg Psychiatry 2012; 83: 371-376.

11 Moher D, Liberati A, Tetzlaff J, et al. Preferred reporting items for systematic reviews and meta-analyses: the PRISMA statement. PLoS Med 2009; 6: e1000097.

12 Centre for Reviews and Dissemination. Centre for Reviews and Dissemination database. 2008. www.crd.york.ac.uk/ crdweb/

13 Higgins J, Green S. Cochrane Handbook for Systematic Reviews of Interventions Version 5.1.0. The Cochrane Collaboration. www.handbook.cochrane.org Date last updated: March 2011.

14 National Institutes of Health. Quality Assessment of Systematic Reviews and Meta-Analyses. www.nhlbi.nih.gov/ health-topics/study-quality-assessment-tools 2014.

15 Critical Appraisal Skills Programme (CASP). Qualitative Research Checklist. https://casp-uk.net/wp-content/ uploads/2018/03/CASP-Qualitative-Checklist-2018_fillable_form.pdf

16 Bertella E, Banfi P, Paneroni M, et al. Early initiation of night-time NIV in an outpatient setting: a randomized non-inferiority study in ALS patients. Eur J Phys Rehabil Med 2017; 53: 892-899.

17 Vrijsen B, Buyse B, Belge C, et al. Randomized cross-over trial of ventilator modes during non-invasive ventilation titration in amyotrophic lateral sclerosis. Respirology 2017; 22: 1212-1218.

18 Crescimanno G, Greco F, Arrisicato S, et al. Effects of positive end expiratory pressure administration during non-invasive ventilation in patients affected by amyotrophic lateral sclerosis: a randomized crossover study. Respirology 2016; 21: 1307-1313.

19 Lopes de Almeida JP, Pinto A, Pinto S, et al. Economic cost of home-telemonitoring care for BiPAP-assisted ALS individuals. Amyotroph Lateral Scler 2012; 13: 533-537.

20 Aboussouan LS, Khan SU, Banerjee M, et al. Objective measures of the efficacy of noninvasive positive-pressure ventilation in amyotrophic lateral sclerosis. Muscle Nerve 2001; 24: 403-409.

21 Lo Coco D, Marchese S, Pesco MC, et al. Noninvasive positive-pressure ventilation in ALS: predictors of tolerance and survival. Neurology 2006; 67: 761-765.

22 Martínez D, Sancho J, Servera E, et al. Tolerance of volume control noninvasive ventilation in subjects with amyotrophic lateral sclerosis. Respir Care 2015; 60: 1765-1771.

23 Mustfa N, Walsh E, Bryant V, et al. The effect of noninvasive ventilation on ALS patients and their caregivers. Neurology 2006; 66: 1211-1217.

24 Vandenberghe N, Vallet A-E, Petitjean T, et al. Absence of airway secretion accumulation predicts tolerance of noninvasive ventilation in subjects with amyotrophic lateral sclerosis. Respir Care 2013; 58: 1424-1432.

25 Servera E, Sancho J, Bañuls P, et al. Bulbar impairment score predicts noninvasive volume-cycled ventilation failure during an acute lower respiratory tract infection in ALS. J Neurol Sci 2015; 358: 87-91.

26 Sheers N, Berlowitz DJ, Rautela L, et al. Improved survival with an ambulatory model of non-invasive ventilation implementation in motor neuron disease. Amyotroph Lateral Scler Frontotemporal Degener 2014; 15: $180-184$

27 Volanti P, Cibella F, Sarvà M, et al. Predictors of non-invasive ventilation tolerance in amyotrophic lateral sclerosis. J Neurol Sci 2011; 303: 114-118.

28 Kareus SA, Kagebein S, Rudnicki SA. The importance of a respiratory therapist in the ALS clinic. Amyotroph Lateral Scler 2008; 9: 173-176.

29 Chiò A, Calvo A, Moglia C, et al. Non-invasive ventilation in amyotrophic lateral sclerosis: a 10 year population based study. J Neurol Neurosurg Psychiatry 2012; 83: 377-381.

30 Ritsma BR, Berger MJ, Charland DA, et al. NIPPV: prevalence, approach and barriers to use at Canadian ALS centres. Can J Neurol Sci 2010; 37: 54-60.

31 Vitacca M, Vianello A. Respiratory outcomes of patients with amyotrophic lateral sclerosis: an Italian nationwide survey. Respir Care 2013; 58: 1433-1441.

32 Chiò A, Bottacchi E, Buffa C, et al. Positive effects of tertiary centres for amyotrophic lateral sclerosis on outcome and use of hospital facilities. J Neurol Neurosurg Psychiatry 2006; 77: 948-950.

33 Chiò A, Silani V. Amyotrophic lateral sclerosis care in Italy: a nationwide study in neurological centers. J Neurol Sci 2001; 191: 145-150.

34 Nixon I, Popkiewicz F, Banerjee S, et al. Using a joint approach to non-invasive ventilation in motor neurone disease. Eur J Palliat Care 2015; 22: 182-184. 
35 Heiman-Patterson TD, Cudkowicz ME, De Carvalho M, et al. Understanding the use of NIV in ALS: results of an international ALS specialist survey. Amyotroph Lateral Scler Frontotemporal Degener 2018; 19: 331-341.

36 Martin NH, Lawrence V, Murray J, et al. Decision making about gastrostomy and noninvasive ventilation in amyotrophic lateral sclerosis. Qual Health Res 2016; 26: 1366-1381.

37 Vrijsen B, Buyse B, Belge C, et al. Noninvasive ventilation improves sleep in amyotrophic lateral sclerosis: a prospective polysomnographic study. J Clin Sleep Med 2015; 11: 559-566.

38 Vrijsen B, Testelmans D, Belge C, et al. Patient-ventilator asynchrony, leaks and sleep in patients with amyotrophic lateral sclerosis. Amyotroph Lateral Scler Frontotemporal Degener 2016; 17: 343-350.

39 Schellhas V, Glatz C, Beecken I, et al. Upper airway obstruction induced by non-invasive ventilation using an oronasal interface. Sleep Breath 2018; 22: 781-788.

40 Bédard M-E, McKim DA. Daytime mouthpiece for continuous noninvasive ventilation in individuals with amyotrophic lateral sclerosis. Respir Care 2016; 61: 1341-1348.

41 Boentert M, Brenscheidt I, Glatz C, et al. Effects of non-invasive ventilation on objective sleep and nocturnal respiration in patients with amyotrophic lateral sclerosis. J Neurol 2015; 262: 2073-2082.

42 Vrijsen B, Buyse B, Belge C, et al. Upper airway obstruction during noninvasive ventilation induced by the use of an oronasal mask. J Clin Sleep Med 2014; 10: 1033-1035.

43 Agrafiotis M, Papanikolaou G, Hospital G, et al. Noninvasive ventilation via mouthpiece in a patient with amyotrophic lateral sclerosis: a method to avoid tracheostomy and improve cough efficacy. Pneumon 2017; 30: $31-35$.

44 Belchior I, Gonçalves MR, Winck JC. Continuous noninvasive ventilation delivered by a novel total face mask: a case series report. Respir Care 2012; 57: 449-453.

45 Diaz-Abad M, Brown JE. Use of volume-targeted non-invasive bilevel positive airway pressure ventilation in a patient with amyotrophic lateral sclerosis. J Bras Pneumol 2014; 40: 443-447.

46 Baxter SK, Baird WO, Thompson S, et al. The initiation of non-invasive ventilation for patients with motor neuron disease: patient and carer perceptions of obstacles and outcomes. Amyotroph Lateral Scler Frontotemporal Degener 2013; 14: 105-110.

47 Sundling I-M, Ekman S-L, Weinberg J, et al. Patients' with ALS and caregivers' experiences of non-invasive home ventilation. Adv Physiother 2009; 11: 114-120.

48 Georges M, Attali V, Golmard JL, et al. Reduced survival in patients with ALS with upper airway obstructive events on non-invasive ventilation. J Neurol Neurosurg Psychiatry 2016; 87: 1045-1050.

49 Nicholson TT, Smith SB, Siddique T, et al. Respiratory pattern and tidal volumes differ for pressure support and volume-assured pressure support in amyotrophic lateral sclerosis. Ann Am Thorac Soc 2017; 14: 1139-1146.

50 Veldhuis SK, Doff MH, Stegenga B, et al. Oral appliance to assist non-invasive ventilation in a patient with amyotrophic lateral sclerosis. Sleep Breath 2015; 19: 61-63.

51 Park D. Application of different ventilator modes in patients with amyotrophic lateral sclerosis according to certain clinical situations: a case report. Medicine 2017; 96: e7899.

52 Hannan LM, Rautela L, Wilson DL, et al. Altering ventilator inspiratory time can reduce autocycling during sleep. Sleep Med 2015; 16: 1301-1303.

53 Yamada S, Nishimiya J, Kurokawa K, et al. Bilevel nasal positive airway pressure and ballooning of the stomach. Chest 2001; 119: 1965-1966.

54 Ando H, Chakrabarti B, Angus RM, et al. Experience of long-term use of non-invasive ventilation in motor neuron disease: an interpretative phenomenological analysis. BMJ Support Palliat Care 2014; 4: 50-56.

55 Ando H, Williams C, Angus RM, et al. Why don't they accept non-invasive ventilation?: insight into the interpersonal perspectives of patients with motor neurone disease. Br J Health Psychol 2015; 20: 341-359.

56 Bach JR. Amyotrophic lateral sclerosis: prolongation of life by noninvasive respiratory aids. Chest 2002; 122 : 92-98.

57 Bach JR, Bianchi C, Aufiero E. Oximetry and indications for tracheotomy for amyotrophic lateral sclerosis. Chest 2004; 126: 1502-1507.

58 Khamankar N, Coan G, Weaver B, et al. Associative increases in amyotrophic lateral sclerosis survival duration with non-invasive ventilation initiation and usage protocols. Front Neurol 2018; 9: 578.

59 Cooper-Knock J, Ahmedzai SH, Shaw P. The use of subcutaneous glycopyrrolate in the management of sialorrhoea and facilitating the use of non-invasive ventilation in amyotrophic lateral sclerosis. Amyotroph Lateral Scler 2011; 12: 464-465.

60 Tamplin J, Baker F, Davies R, et al. Exploring the feasibility of a music-assisted relaxation intervention to assist with the initiation of non-invasive ventilation in people with motor neuron disease. Music Med 2017; 9: 86-97.

61 Gruis KL, Brown DL, Lisabeth LD, et al. Longitudinal assessment of noninvasive positive pressure ventilation adjustments in ALS patients. J Neurol Sci 2006; 247: 59-63.

62 Oliver D, Banerjee S, Vincent-Smith L, et al. The use of a hand held ventilator to supplement NIV for patients with ALS/MND with respiratory insufficiency. Amyotroph Lateral Scler Frontotemporal Degener 2018; 19: 313-314.

63 Butz M, Wollinsky KH, Wiedemuth-Catrinescu U, et al. Longitudinal effects of noninvasive positive-pressure ventilation in patients with amyotrophic lateral sclerosis. Am J Phys Med Rehabil 2003; 82: 597-604.

64 Farrero E, Prats E, Povedano M, et al. Survival in amyotrophic lateral sclerosis with home mechanical ventilation: the impact of systematic respiratory assessment and bulbar involvement. Chest 2005; 127: 2132-2138.

65 Bourke SC, Bullock RE, Williams TL, et al. Noninvasive ventilation in ALS: indications and effect on quality of life. Neurology 2003; 61: 171-177.

66 Jackson CE, Lovitt S, Gowda N, et al. Factors correlated with NPPV use in ALS. Amyotroph Lateral Scler 2006; 7: $80-85$.

67 De Vito EL, Suárez AA, Monteiro SG. The use of full-setting non-invasive ventilation in the home care of people with amyotrophic lateral sclerosis-motor neuron disease with end-stage respiratory muscle failure: a case series. J Med Case Rep 2012; 6: 42.

68 Berlowitz DJ, Howard ME, Fiore JF Jr, et al. Identifying who will benefit from non-invasive ventilation in amyotrophic lateral sclerosis/motor neurone disease in a clinical cohort. J Neurol Neurosurg Psychiatry 2015; 87: $280-286$. 
Lemoignan J, Ells C. Amyotrophic lateral sclerosis and assisted ventilation: how patients decide. Palliat Support Care 2010; 8: 207-213.

70 Aridegbe T, Kandler R, Walters SJ, et al. The natural history of motor neuron disease: assessing the impact of specialist care. Amyotroph Lateral Scler Frontotemporal Degener 2013; 14: 13-19.

71 Traynor BJ, Alexander M, Corr B, et al. Effect of a multidisciplinary amyotrophic lateral sclerosis (ALS) clinic on ALS survival: a population based study, 1996-2000. J Neurol Neurosurg Psychiatry 2003; 74: 1258-1261.

72 Rooney J, Byrne S, Heverin M, et al. A multidisciplinary clinic approach improves survival in ALS: a comparative study of ALS in Ireland and Northern Ireland. J Neurol Neurosurg Psychiatry 2015; 86: 496-501.

73 Rodríguez de Rivera FJ, Oreja Guevara C, Sanz Gallego I, et al. Outcome of patients with amyotrophic lateral sclerosis attending in a multidisciplinary care unit. Neurología 2011; 26: 455-460.

74 Chatwin M, Nickol AH, Morrell MJ, et al. Randomised trial of inpatient versus outpatient initiation of home mechanical ventilation in patients with nocturnal hypoventilation. Respir Med 2008; 102: 1528-1535.

75 Hazenberg A, Kerstiens HAM, Prins SCL, et al. Initiation of home mechanical ventilation at home: a randomised controlled trial of efficacy, feasibility and costs. Respir Med 2014; 108: 1387-1395.

76 Berry RB, Chediak A, Brown LK, et al. Best clinical practices for the sleep center adjustment of noninvasive positive pressure ventilation (NPPV) in stable chronic alveolar hypoventilation syndromes. J Clin Sleep Med 2010; 6: 491-509.

77 Chatwin M, Toussaint M, Gonçalves MR, et al. Airway clearance techniques in neuromuscular disorders: a state of the art review. Respir Med 2018; 136: 98-110.

78 Janssens J-P, Borel J-C, Pépin J-L. Nocturnal monitoring of home non-invasive ventilation: the contribution of simple tools such as pulse oximetry, capnography, built-in ventilator software and autonomic markers of sleep fragmentation. Thorax 2011; 66: 438-445.

79 Fox N, Hirsch-Allen AJ, Goodfellow E, et al. The impact of a telemedicine monitoring system on positive airway pressure adherence in patients with obstructive sleep apnea: a randomized controlled trial. Sleep 2012; 35: 477-481.

80 Hobson EV, Baird WO, Cooper CL, et al. Using technology to improve access to specialist care in amyotrophic lateral sclerosis: a systematic review. Amyotroph Lateral Scler Frontotemporal Degener 2016; 17: 313-324.

81 Volpato E, Banfi P, Pagnini F. A psychological intervention to promote acceptance and adherence to non-invasive ventilation in people with chronic obstructive pulmonary disease: study protocol of a randomised controlled trial. Trials 2017; 18: 59. 